\title{
Correction: Zhang, M., et al. Estimation of Vegetation Productivity Using a Landsat 8 Time Series in a Heavily Urbanized Area, Central China. Remote Sens. 2019, 11, 133
}

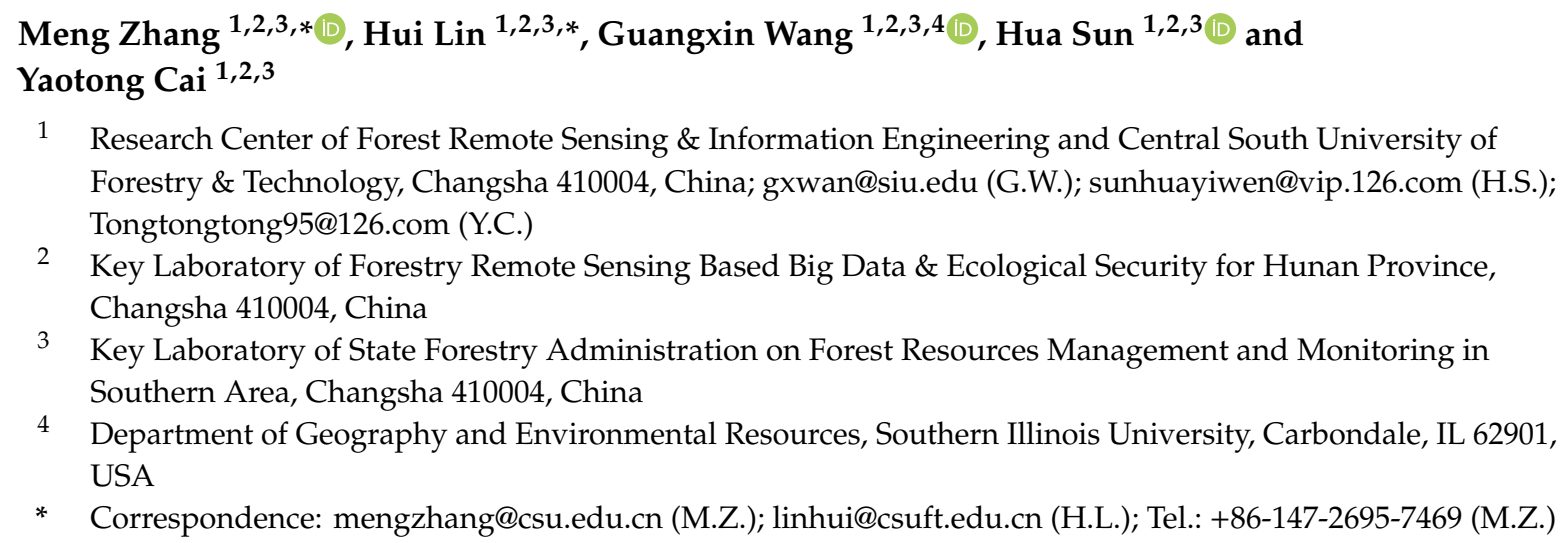

The authors wish to make the following corrections to this paper [1]:

\section{Change in Author Names (Withdraw one author)}

In the original version of our article [1], Guangxing Wang was listed as a co-author. However, we discovered that we had misspelled Guangxing Wang's email address, so that he was totally unaware of this paper. Thus, we would like to request to withdraw Guangxing Wang from this paper. Therefore, the list of the authors and their corresponding affiliations have been changed as follows:

$$
\text { Meng Zhang } 1,2,3, * \text {, Hui Lin } 1,2,3, * \text {, Hua Sun }{ }^{1,2,3} \text { and Yaotong Cai }{ }^{1,2,3}
$$

1 Research Center of Forest Remote Sensing \& Information Engineering, Central South University of Forestry \& Technology, Changsha 410004, China

2 Key Laboratory of Forestry Remote Sensing Based Big Data \& Ecological Security for Hunan Province, Changsha 410004, China

3 Key Laboratory of State Forestry Administration on Forest Resources Management and Monitoring in Southern Area, Changsha 410004, China

The authors would like to apologize for any inconvenience caused to the readers by these changes.

\section{Reference}

1. Zhang, M.; Lin, H.; Guangxing, W.; Sun, H.; Cai, Y. Estimation of Vegetation Productivity Using a Landsat 8 Time Series in a Heavily Urbanized Area, Central China. Remote Sens. 2019, 11, 133. [CrossRef] 